\title{
El nematodo Caenorhabditis elegans como modelo para evaluar el potencial antihelmíntico de extractos de plantas
}

\section{The nematode Caenorhabditis elegans as a model to assess the anthelmintic potential from plant extracts}

\author{
Zyanya Mayoral Peñaa , Denia María Piña Vazquez ${ }^{a}$, Maricela Gómez Sánchez ${ }^{a}$, Luis Antonio Salazar Olivob, \\ Gabriela Aguilar Tipacamúa, Fausto Arellano Carbajala*
}

\begin{abstract}
RESUMEN
Los nematodos parásitos afectan la productividad pecuaria, dando lugar a pérdidas económicas considerables. Sin embargo, son pocos los antihelmínticos disponibles en el mercado y la resistencia de los parásitos a los existentes va en incremento, por lo que su control es actualmente ineficiente. En este sentido, la búsqueda de nuevas estrategias para controlar la parasitosis es una necesidad actual. Una posibilidad para el control estriba en la investigación de metabolitos derivados de plantas, ya que el conocimiento sobre su uso tradicional es un referente de inicio. Sin embargo, la eficacia antihelmíntica, así como los mecanismos de acción de plantas con uso tradicional como antiparasitarios, poco se han investigado científicamente, y en la mayoría de los casos se desconocen los compuestos activos responsables del efecto anti-parasitario. Una de las limitantes del estudio experimental del potencial antihelmíntico es la dificultad de trabajar con modelos parásitos. En este sentido, el nematodo de vida libre $C$. elegans es un modelo útil para entender el mecanismo de acción de drogas antihelmínticas. Por un lado, este nematodo se puede mantener y manipular en laboratorio con facilidad; por otro, comparte características fisiológicas y homología genética con nematodos parásitos, lo que permite comparaciones válidas. En esta revisión se pretende mostrar el potencial de la búsqueda de compuestos activos derivados de plantas tradicionalmente utilizadas como antiparasitarios como punto de partida en el desarrollo de nuevos fármacos antihelmínticos, así como resaltar las ventajas que $C$. elegans proporciona para entender los mecanismos de acción de nuevos compuestos.
\end{abstract}

PALABRAS CLAVE: Resistencia, Antihelmínticos, Caenorhabditis elegans, Extractos de plantas, Conocimiento tradicional.

\section{ABSTRACT}

Parasitic nematodes affect livestock productivity, leading to economic losses. Despite their importance, few anthelmintics have been developed and several parasites have developed resistance to them, rendering control of parasitic infections very inefficient. In this sense, the search for new strategies to control parasitic nematodes is timely and relevant. One possibility is to conduct research on metabolites derived from plants with anthelmintic potential, since knowledge about its traditional use is a good starting point. However, the anthelmintic efficacy and mechanisms of action of plants traditionally used, have been scarcely investigated, and the bioactive compounds responsible for the effects remain mostly unknown. One of the limitations that experimental studies on potential anthelmintic face is the difficulty of working with parasite models. In this respect, the free-living nematode $C$. elegans is a useful tool for understanding the mechanism of action of anthelmintic drugs as well as mechanisms of resistance. First, $C$. elegans can be maintained and easily manipulated in the laboratory; in addition, C. elegans shares physiological characteristics and has genetic homology with nematode parasites, allowing valid comparisons. This review aims to underscore the potential of finding active compounds derived from plants traditionally used as anthelmintic as a starting point in the development of new drugs, as well as highlight the benefits that $C$. elegans provides for understanding mechanisms of action of novel anthelmintic.

KEY WORDS: Resistance, Anthelmintics, Caenorhabditis elegans, Plant extracts, Traditional knowledge.

Recibido el 8 de octubre de 2015. Aceptado el 8 de marzo de 2016.

${ }^{a}$ Facultad de Ciencias Naturales, Universidad Autónoma de Querétaro. Av. de las Ciencias S/N. 76230. Querétaro, Querétaro, México.

${ }^{\mathrm{b}}$ Instituto Potosino de Investigación Científica y Tecnológica. San Luis Potosí S.L.P., México.

*Autor de correspondencia: fausto.arellano@uaq.mx.

Origen del apoyo recibido: Fondo para el Fortalecimiento de la Investigación UAQ-FNB-2012-11 (20101169). 


\section{INTRODUCCIÓN}

Los nematodos parásitos impactan negativamente a la salud humana y al sector pecuario. La mayoría de los animales destinados para consumo experimentan una exposición constante o estacional a parásitos a lo largo de su vida, lo cual frecuentemente genera importantes pérdidas económicas ${ }^{(1)}$.

El control de las parasitosis mediante antihelmínticos sintéticos de uso comercial es actualmente ineficiente. Esto se debe principalmente a que los nematodos que parasitan a especies de importancia económica han desarrollado resistencia hacia las principales familias de fármacos antiparasitarios de amplio espectro ${ }^{(2)}$. La resistencia puede ocurrir a varios niveles; por un lado, puede deberse a cambios en el blanco molecular, de modo que el fármaco no reconoce el objetivo y por tanto se vuelve ineficaz; o bien, se dan modificaciones metabólicas en el nematodo que inactivan o eliminan el fármaco ${ }^{(3)}$. Pese a la resistencia, el desarrollo de nuevas clases de antihelmínticos es lento e ineficiente. Por tal razón es necesaria la generación de nuevos fármacos, así como métodos que nos permitan comprender sus mecanismos de acción $n^{(4)}$.

Una alternativa metodológica para el desarrollo de nuevos fármacos es la búsqueda de plantas con potencial antihelmíntico, con base en el conocimiento y prácticas tradicionales ${ }^{(5)}$. En países en desarrollo, la etnobotánica forma parte importante de la cultura y de la medicina preventiva, ya que existen zonas que carecen de acceso a la medicina convencional para el cuidado de la salud $^{(6)}$. La evidencia de las propiedades antihelmínticas de extractos de plantas proviene principalmente de fuentes etnobotánicas, documentadas en diferentes partes del mundo ${ }^{(7-13)}$.

A pesar de este potencial, la actividad antihelmíntica de muchas plantas utilizadas localmente por diversas culturas, no se ha corroborado experimentalmente, y en la mayoría de los casos se desconoce cuáles son los metabolitos activos y sus mecanismos de acción. Por esto, existe interés científico en el estudio de las prácticas etnobotánicas, para el desarrollo y promoción de medicamentos eficaces, basados en los principios activos de plantas disponibles a nivel local(8-13).
En esta revisión se resalta la relevancia de evaluar la capacidad antihelmíntica de las plantas que el conocimiento tradicional reporta, y se discute el valor del nematodo Caenorhabditis elegans como modelo de estudio para determinar la capacidad antihelmíntica de las plantas.

\section{El impacto de los helmintos en la producción animal}

La principal causa de pérdidas económicas relacionadas con la producción de rumiantes a nivel mundial es el parasitismo ${ }^{(14)}$; para contrarrestarlo, se han implementado diferentes estrategias, tales como el control biológico y la selección de animales resistentes. Para el tratamiento y el control de las helmintiasis se depende críticamente de la eficacia de los fármacos antihelmínticos; sin embargo, su utilidad ha disminuido severamente por la rápida evolución y propagación de la resistencia de los helmintos ${ }^{(15)}$.

Además, las pérdidas económicas pecuarias ocasionadas por la exposición constante a parásitos en los animales destinados para consumo son altas, y ocurren principalmente en países en vías de desarrollo. Se estima que las pérdidas han llegado a alcanzar cifras de 1.7 billones de dólares anuales ${ }^{(16)}$. La intensificación de los sistemas de producción animal ha dado lugar a una dependencia casi exclusiva de los fármacos antiparasitarios, por lo que el desarrollo de resistencia a la acción de los principales fármacos antihelmínticos de amplio espectro, es una seria amenaza para los sistemas de producción animal(17). Desgraciadamente, la industria farmacéutica tiene poco interés en invertir en el desarrollo de antihelmínticos, debido a que la producción de dichos fármacos representa un mercado inseguro y no muy rentable, en particular porque está dirigido principalmente a países en vías de desarrollo(18). De hecho, se estima que la elaboración de nuevos antihelmínticos implicaría la inversión de 200 millones de dólares y 10 años de investigación para ingresar al mercado veterinario(19). En este contexto, la búsqueda de recursos biológicos locales como plantas, representa una fuente alternativa de metabolitos con potencial antihelmíntico. 


\section{La resistencia a los antihelmínticos}

El manejo pecuario actual incluye el uso indiscriminado de fármacos antiparasitarios, principalmente en cuanto a la administración masiva de medicamentos antihelmínticos ${ }^{(20-23)}$, acción respaldada por la Organización Mundial de la Salud (OMS) en su plan de trabajo (2015-2020) sobre el control y eliminación de las enfermedades tropicales desatendidas(24-25). Esta estrategia conlleva a la generación de resistencia por parte de los helmintos.

La resistencia se presenta cuando dentro de una población existe una frecuencia alta de helmintos capaces de tolerar dosis de un compuesto dado, lo que una población normal de la misma especie no toleraría. El hecho de que dicha capacidad sea heredable determina el impacto de la resistencia ${ }^{(26)}$. En realidad, la resistencia a los antihelmínticos ocurre como una respuesta a las presiones de selección que ejercen los fármacos sobre las poblaciones de parásitos que infectan a un organismo. De hecho, suele presentarse de forma simultánea en los nematodos parásitos, los cuales dejan de ser sensibles al efecto de varias drogas ${ }^{(27)}$.

A comienzos de 1960 por primera vez se reportó resistencia antihelmíntica a la droga fenotiazina por parte del parásito gástrico hematófago Haemonchus contortus $^{(28)}$ y después en pequeños estróngilos típicos del caballo(29). En 1961 se liberó el tiabendazol en el mercado, un fármaco anunciado como el primero en combinar una eficacia nematicida de amplio espectro y una baja toxicidad. Sin embargo, a los pocos años de uso se reportó la resistencia al tiabendazol por parte de $\boldsymbol{H}$. contortus, Teladorsagia (Ostertagia) y Trichostrongy/us ${ }^{(30)}$. Para mediados de 1970, se encontró que múltiples especies presentaban resistencia a los benzimidazoles, y que ésta era común y generalizada en ovejas y caballos en todo el mundo. El mismo patrón se repitió en la década de los ochenta con otros fármacos, como el imidazotiazoltetrahidropirimidina y la avermectinamilbemicina(31,32,33). Para la década de 1990, la resistencia antihelmíntica ya era un problema generalizado. Los informes de helmintos resistentes a múltiples fármacos aumentaron, por lo que el fenómeno pasó a ser percibido como una amenaza importante para la producción de pequeños rumiantes en varias partes del mundo(34) como África, Argentina, Paraguay, Brasil y Uruguay, donde cabras y borregos fueron tratados durante un breve periodo de tiempo con tiabendazol y se observó el desarrollo de resistencia rápida a las tres principales clases de antihelmínticos ${ }^{(35)}$.

Es importante mencionar que los países industrializados no son excepción a la generación de resistencia a antihelmínticos, puesto que también hay registros de resistencia a más de siete fármacos anti-parasitarios en Australia, España y Holanda(36,37,38). Por mencionar solamente a un parásito muy estudiado, $H$. contortus, éste ha desarrollado tal resistencia a los antihelmínticos existentes, que sus infecciones amenazan la viabilidad de las industrias de los pequeños rumiantes en gran parte de América del Sur $(39,40,41)$, Sudáfrica ${ }^{(42)}$, Malasia ${ }^{(43,44)}$ y el sureste de los Estados Unidos ${ }^{(45)}$.

\section{Mecanismos de resistencia}

La resistencia a los fármacos antihelmínticos no surge de manera espontánea en un parásito, sino que se adquiere con el tiempo, cuando una droga que es inicialmente efectiva para un fin terapéutico determinado, deja de serlo. Actualmente la resistencia se presenta hacia los tres principales grupos de fármacos antihelmínticos: 1) benzimidazoles, 2) imidazotiazoles y tetrahidropirimidinas, y 3) avermectinas y milbemicinas, mismos que se describen de manera general a continuación.

Los compuestos benzimidazoles (BZD) actúan ligándose selectivamente a la subunidad $\beta$ de la proteína tubulina de nematodos y cestodos, lo que modifica el patrón de polimerización para la formación de microtúbulos. La resistencia ocurre cuando los genes que codifican para $\beta$ tubulina sufren mutaciones, lo cual reduce la afinidad del receptor por $B Z D^{(46,47)}$.

Los imidazotiazoles (levamisol) y tetrahidropirimidinas (p. ej.: morantel y pirantel), actúan rápido y selectivamente como agonistas colinérgicos sobre receptores nicotínicos sinápticos y extrasinápticos de las membranas de las células musculares de los nematodos ${ }^{(48,49)}$. Cuando el 
levamisol se une a los receptores nicotínicos se abren los canales iónicos y aumenta la conductancia al sodio, por lo que son despolarizadas las membranas de las células musculares, resultando en contracción muscular y parálisis espástica ${ }^{(48)}$. La resistencia a estos agonistas colinérgicos se produce por cambios en las propiedades de la población de receptores nicotínicos ${ }^{(50)}$ y a través de una alteración en la unión de estos fármacos con sus receptores nicotínicos. Se ha determinado que los genes lev-1, unc-29 y unc-38 son algunos de los genes responsables de la resistencia, ya que codifican subunidades proteicas que forman los canales iónicos nicotínicos del nematodo y afectan la afinidad del fármaco por el canal(51).

Las avermectinas (AVM) y milbemicinas (MBM) actúan como agonistas de alta afinidad sobre la subunidad a de canales iónicos selectivos a cloro, mismos que están presentes en nematodos y

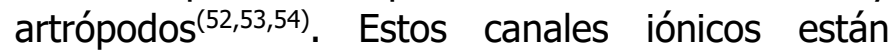
compuestos por cinco subunidades proteicas que se combinan entre sí para formar un pentámero(53,54,55). Cuando AVM y MBM se unen selectiva e irreversiblemente a estos receptores, aumenta la permeabilidad de la membrana al cloro, lo cual origina la hiperpolarización de la membrana de la célula muscular o neuronal del parásito. La resistencia a AVM y MBM podría entonces estar asociada a modificaciones en las subunidades del receptor $\mathrm{GluCl}$, o a la expresión aumentada de una glicoproteína de membrana (glicoproteína P), lo que posiblemente impediría alcanzar concentraciones activas de la molécula antiparasitaria en el receptor de glutamato del parásito resistente ${ }^{(56,57,58)}$.

\section{Plantas con capacidad antihelmíntica}

La tasa de diseño y elaboración de fármacos activos nuevos no es suficiente para las necesidades actuales ${ }^{(59)}$, y no existe información pública disponible sobre los planes para el desarrollo de nuevas drogas antihelmínticas. Recurrir al conocimiento etnofarmacológico es una manera de comenzar la búsqueda de nuevos compuestos con potencial antiparasitario ${ }^{(2)}$, que tengan mecanismos de acción distintos a los descritos hasta el momento.
Las plantas son una fuente de biocompuestos, algunos con potencial para tratar infecciones parasitarias de animales de interés pecuario( ${ }^{(2)}$. Sin embargo, la evidencia científica sobre la eficacia antiparasitaria de extractos botánicos es limitada, independientemente de su amplio uso tradicional. Por esto, su validación es muy necesaria antes de adoptarlas como un nuevo método de tratamiento ${ }^{(60,61)}$.

La evidencia de las propiedades antihelmínticas de extractos de plantas se deriva principalmente de fuentes etnoveterinarias, conocimiento que se ha documentado en diferentes partes del mundo ${ }^{(8-10,14,15)}$. En Reino Unido, por ejemplo, desde principios del siglo $\mathrm{XX}$, las hojas y flores secas del epazote, Chenopodium ambrosioides, se han utilizado como antihelmínticos ${ }^{(62)}$. El epazote todavía se utiliza para el tratamiento de infecciones por helmintos en América Latina. Asimismo, el helecho macho Dryopteris filix-mas y la planta conocida como ajenjo o hierba santa (género Artemisia) se usan contra diversos nematodos que afectan a las aves de corral(62). En los países en vías de desarrollo se tienen identificadas varias plantas que tienen el potencial de ser utilizadas como antihelmínticos ${ }^{(63)}$.

La mayoría de los datos recogidos por fuentes etnoveterinarias son descriptivos y no indagan en los mecanismos de acción ni en los compuestos activos $^{(64)}$. Los métodos de preparación y posología de algunas de las plantas que se utilizan como antiparasitarios se han documentado en algunos

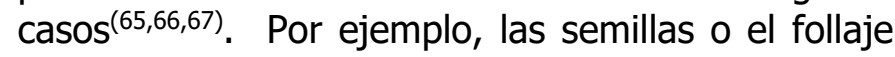
del ajo, Allium sativum, A. cepa L.; la menta, Mentha piperita L.; la nuez pecana, Carya illinoinensis, el eneldo, Anethum graveolens L.; y el perejil, Petroselinum crispum Miller, se utilizan para tratar animales que sufren de parasitismo gastrointestinal, mientras que el pepino, Cucumis sativus, y las semillas de la calabaza, Cucurbita maxima, se utilizan para expulsar tenias( ${ }^{(68)}$. En ninguno de los casos se ha realizado trabajo experimental para determinar y comprobar la eficacia antihelmíntica, así como su mecanismo de acción ${ }^{(61)}$.

Una vez detectadas las plantas con potencial antihelmíntico, se requiere detectar y cuantificar a los posibles compuestos activos, para validar su 
potencial antihelmíntico y finalmente entender sus mecanismos de acción sobre el parásito. Con base en los extractos botánicos, pueden identificarse plantas que tienen potencial para ser utilizadas como base para la elaboración de fármacos antihelmínticos complementarios a los existentes ${ }^{(63)}$.

La mayoría de los ensayos que evalúan el potencial antihelmíntico de extractos vegetales se enfocan en estudiar el efecto de los extractos sobre la fertilidad y motilidad de sus modelos de estudio $^{(69,70)}$. En estos casos se determina la presencia o ausencia de un efecto y no se indaga más allá. A la fecha se han explorado diferentes métodos para evaluar el potencial antihelmíntico de extractos botánicos tanto in vivo como in vitro. La principal ventaja de los ensayos in vitro es que suelen tener un menor costo que los ensayos con animales hospederos vivos. Además, al hacer estudios in vitro, se puede evaluar un mayor número de plantas y concentraciones, y es posible fraccionar un extracto de interés y probar el o los compuestos purificados. Por estas ventajas los ensayos in vitro se han usado para detectar la actividad antihelmíntica de las plantas ${ }^{(69-72)}$.

Una vez que el extracto ha sido seleccionado se requiere elegir un nematodo para probar el efecto. Pensar en un parásito como organismo modelo es lo primero que viene a la mente; sin embargo, es difícil mantenerlos en cultivos debido a que en su mayoría presentan ciclos de vida complejos, requieren de hospederos intermediarios y condiciones que son difíciles de reproducir en laboratorio. Por lo tanto, una alternativa es el uso de nematodos modelo de vida libre como $C$. elegans ${ }^{(12,65,69,70)}$.

Resulta evidente que no todas las preguntas pueden responderse bajo condiciones de laboratorio, pero poder mantener condiciones específicas y medibles permite responder preguntas puntuales con precisión. En el caso de la búsqueda de extractos de plantas con acción antihelmíntica, se requiere asegurar que el extracto sea responsable de la actividad antiparasitaria, y que el efecto observado no responda a alguna variable ajena al extracto.

\section{C. elegans como modelo para estudios de actividad antihelmíntica}

El nematodo de vida libre $C$. elegans, se ha propuesto como un buen modelo para estudios in vitro, ya que su mantenimiento en laboratorio es fácil y de bajo costo ${ }^{(69)}$. En condiciones de laboratorio, C. elegans se alimenta de cepas no patógenas de Escherichia coli, las cuales son fáciles de mantener en cultivo (Figura 1). Debido a que este nematodo se ha utilizado como modelo animal desde hace mucho tiempo, existen protocolos de manejo y está disponible una cantidad considerable de información sobre la especie en línea(70). A continuación se presentan algunos aspectos básicos de la biología de este nematodo modelo.

A nivel anatómico, el cuerpo de $C$. elegans es transparente (Figura 2), por lo que no requiere métodos de aclaramiento para observar sus estructuras internas en distintos estadios de vida(71). Esta característica podría ser ideal para evaluar el efecto de algún extracto sobre el movimiento faríngeo, como sucede con la ivermectina ${ }^{(72)}$.

C. elegans se desarrolla a temperatura ambiente en un periodo de aproximadamente tres días, en los que pasa de huevo a adulto fértil. Existen

Figura 1. El nematodo de vida libre Caenorhabditis elegans

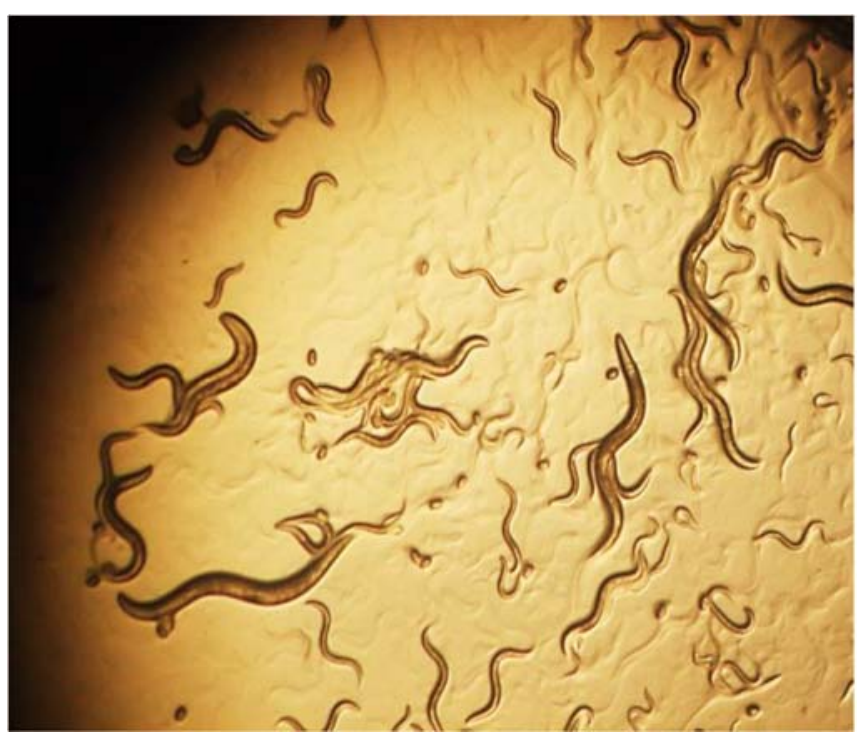

Imaqen tomada por María de Jesús Guerrero Sánchez. 
Figura 2. Cepa silvestre N2 de C. elegans

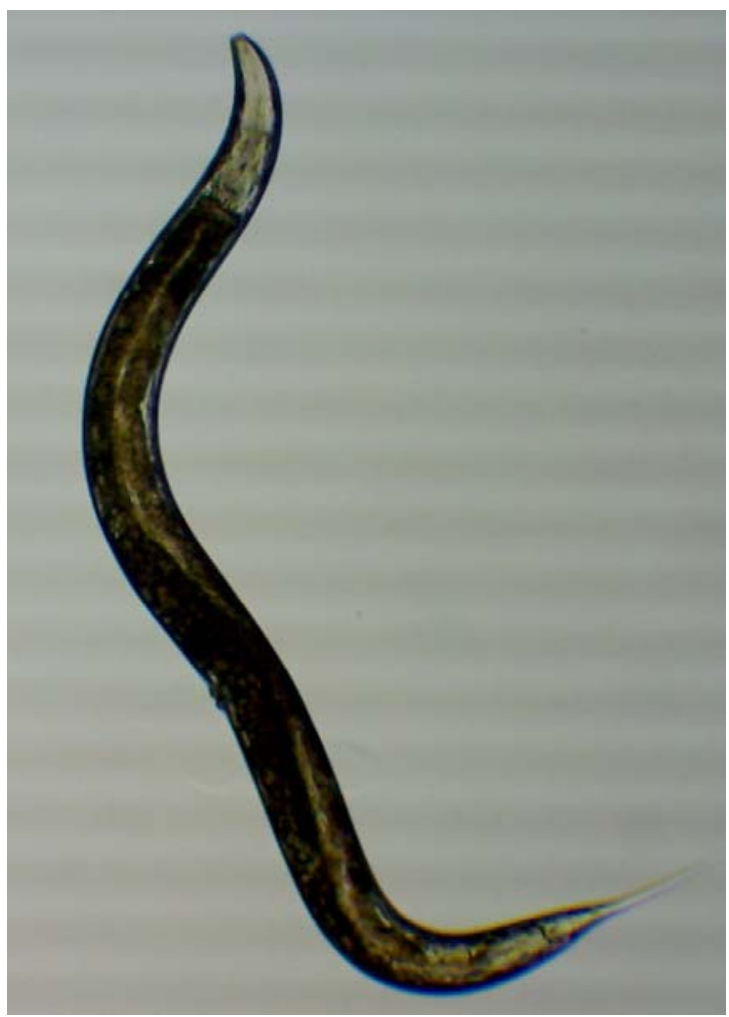

Imagen tomada por Zyanya Mayoral Peña

hermafroditas y machos, y cada hermafrodita puede dejar entre 300 y 1,000 descendientes $^{(71)}$, lo cual es ventajoso a nivel experimental, ya que en poco tiempo se pueden generar cientos de individuos. Para estudios sobre antihelmínticos, cuando se pretende probar extractos de plantas en varias concentraciones, es muy útil contar con muchos individuos en breves periodos de tiempo. Por otro lado, ya que la mayoría de los individuos son hermafroditas que se autofertilizan, se tiene cierta uniformidad genética entre individuos ${ }^{(71,73)}$. Esto implica que si se desea estudiar el mecanismo de acción a nivel genético o molecular, será más sencillo detectarlo, puesto que los individuos serían réplicas experimentales.

Otra característica de particular importancia es que $C$. elegans es el nematodo más estudiado en términos moleculares, de hecho fue el primer metazoario en secuenciarse su genoma ${ }^{(74)}$, y actualmente se conoce la secuencia de cada nucleótido con alto nivel de certeza ${ }^{(75)}$. Si comparamos esta situación con la de los nematodos parásitos, solamente nueve de ellos han sido completamente secuenciados $^{(76)}$. C. elegans va muy adelantado puesto que también su transcriptoma está completamente descrito y disponible (www.nematodes.org). La información genómica y neural disponible en este nematodo ha permitido la aplicación de potentes enfoques genéticos moleculares para entender el modo de acción de antiparasitarios de uso comercial disponibles actualmente, así como los mecanismos de resistencia hacia estos ${ }^{(77)}$. Además, están disponibles varias cepas mutantes y transgénicas de C. elegans, lo que facilita la búsqueda de mecanismos de acción en antihelmínticos comerciales como benzimidazoles, lactonas macrocíclicas y levamisol ${ }^{(54,78)}$. Por otro lado, en $C$. elegans también es posible evaluar el efecto de compuestos con potencial antiparasitario en términos de oviposición ${ }^{(79-82)}$, ya que la fisiología de este proceso está bien descrita para la especie.

El modelo nematodo $C$. elegans es todavía más útil para evaluar el potencial antihelmíntico de un extracto, porque comparte características morfológicas, genéticas y neurológicas con los nematodos parásitos ${ }^{(77,83,84)}$. Incluso se ha reportado que algunos nematodos, como Ascaris suum, que no son tan cercanos filogenéticamente a $C$. elegans, tienen alta similitud en la neuromusculatura y en los neurotransmisores ${ }^{(84,85)}$. Esto es relevante, debido a que el mecanismo de acción de varios de los antihelmínticos implica aspectos relacionados con la motilidad. Hay algunos mecanismos que son compartidos entre nematodos independientemente de sí son parásitos o no, y por ende, los compuestos que actúan sobre ellos actuarán de forma semejante sobre los parásitos. Un ejemplo es el benzimidazol, el cual inhibe la polimerización de la $\beta$-tubulina, que está presente en la mayoría de los nematodos conocidos, por lo que su mecanismo de acción es generalizado sin que importe la distancia

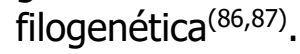

A pesar de que el que algunos mecanismos sean compartidos, es importante aclarar que esto no asegura que un compuesto dado tenga en todos los nematodos parásitos exactamente el mismo mecanismo de acción observado en $C$. elegans. Por 
ejemplo, se ha visto que en nematodos filariales, la diferencia entre genes de parásitos es semejante a la que diferencia a los nematodos parásitos de $C$. elegans $(\geq 30 \%)^{(78)}$. Debido a las presiones de selección a las que están expuestos los nematodos parásitos, estos han desarrollado diversos mecanismos de resistencia, mismos que podrían implicar otras formas de evadir el efecto de los fármacos, tal como la p-glicoproteína, que tiene que ver con mecanismos de desintoxicación ${ }^{(88)}$. En este sentido, es posible que los efectos de los fármacos sobre $C$. elegans podrían expresarse de forma distinta. A pesar de esto, los estudios que utilizan a C. elegans como modelo para ensayos de compuestos anti-parasitarios, podrían avanzar en la detección de moléculas útiles y mecanismos de acción de dichas moléculas.

C. elegans ha ayudado a determinar las propiedades antihelmínticas de distintos extractos de plantas. Extractos acuosos y orgánicos de 17 plantas se seleccionaron para su investigación con base en sus usos tradicionales en África subsahariana para enfermedades gastrointestinales, y se probó su actividad con base en el efecto de la motilidad de $C$. elegans. De estos, ocho mostraron fuerte evidencia de actividad ${ }^{(89,90)}$.

Extractos etanólicos y acuosos de plantas medicinales de Camerún y Ghana fueron probados para determinar su actividad antihelmíntica mediante el uso de $C$. elegans ${ }^{(91)}$. Estos mismos autores encontraron que algunos extractos acuosos y etanólicos de plantas afectaban el crecimiento y supervivencia de $C$. elegans, por lo que sugieren que estos extractos podrían proveer de alternativas en el control de infecciones helmínticas. Con base en el contenido de polifenoles en estos extractos, también probaron el efecto de tres fenoles en cepas silvestres y resistentes a antihelmínticos comerciales de $C$. elegans, lo que les permitió ver que el ácido elágico era una opción potencial para el tratamiento de infecciones por nematodos, incluso en casos de resistencia a antihelmínticos comerciales ${ }^{(92)}$.

Katiki et $a^{\left({ }^{93)}\right.}$ probaron diferentes extractos de plantas en $C$. elegans y encontraron que plantas ricas en taninos hidrolizables tienen mayor actividad antihelmíntica que plantas ricas en taninos condensados. $C$. elegans también ha permitido probar la mejor opción de solvente para preparar los extractos $^{(94,95)}$. Además, $C$. elegans ha sido probado para identificar metabolitos secundarios de hongos con propiedades antihelmínticas ${ }^{(96)}$. Igualmente, la búsqueda de nuevos compuestos antihelmínticos también puede verse acelerada mediante la implementación de sistemas automatizados para detectar efectos en el movimiento y desarrollo de $C$. elegans $^{(97)}$. C. elegans también ha sido utilizado para determinar interacciones sinérgicas entre antihelmínticos comerciales; esta estrategia es una alternativa contra el incremento de resistencia a antihelmínticos individuales ${ }^{(98,99)}$.

Análisis genético en $C$. elegans ha ayudado a identificar los mecanismos de acción de algunos nuevos antihelminticos; como la betaina que actúa sobre un canal iónico activado por ligando ${ }^{(100)}$ y la emodepsida que paraliza los músculos de la pared del cuerpo y la faringe, vía un mecanismo que involucra a receptores tipo latrofilina ${ }^{(101-102)}$.

Burns et $a{ }^{(103)}$ analizaron 67,012 compuestos para identificar aquéllos que afectaban la supervivencia de $C$. elegans, los compuestos seleccionados los vuelven a probar y seleccionan los que afectan a dos especies de nematodos parásitos y que no afectan a dos modelos vertebrados; finalmente identifican 30 moléculas con potencial antihelmíntico y después determinan de éstas, cuáles tienen la mayor probabilidad de generar resistencia en un periodo menor de tiempo. Estos trabajos, entre otros, han ayudado a establecer a $C$. elegans como un sistema modelo para el descubrimiento de antihelmínticos.

\section{CONCLUSIÓN}

Un buen modelo no sólo es aquel que permite extrapolar, sino aquel que además da lugar al diseño de experimentos que llevan a responder nuevas preguntas. Para el caso particular del escrutinio de extractos de plantas, $C$. elegans permite diseñar ensayos que van más allá de contar el número de huevos del nematodo y observar el efecto sobre la motilidad.

El potencial antihelmíntico de la mayoría de las plantas utilizadas como antiparasitarias en medicina tradicional en México es un campo abierto para la 
exploración científica. Indagar en los mecanismos de acción de los compuestos potenciales como antihelmínticos será importante para buscar alternativas a la resistencia. El nematodo modelo $C$. elegans, representa una alternativa útil para explorar el mecanismo de acción de los compuestos de las plantas que poseen un probable efecto antiparasitario. Debido a que hasta el momento es el nematodo mejor estudiado en términos moleculares y fisiológicos, se puede indagar sobre la interacción de los metabolitos activos presentes en las plantas a nivel genético y molecular. La evaluación de metabolitos potencialmente antihelmínticos, procedentes de fuentes naturales, es una de las alternativas que se investiga como solución a la problemática de la generación de resistencia hacia los fármacos antiparasitarios comercialmente disponibles. En conjunto, el uso del nematodo modelo $C$. elegans para explorar los mecanismos de acción de compuestos de plantas con potencial antiparasitario es un paso más para intentar resolver los problemas de parasitosis que aquejan a países en vías de desarrollo.

\section{AGRADECIMIENTOS}

Se agradece el apoyo financiero por parte de FOFI-UAQ (FNB-2012-11 20101169).

\section{LITERATURA CITADA}

1. Hotez PJ, Brindley PJ, Bethony JM, King CH, Pearce EJ, Jacobson J. Helminth infections: the great tropical diseases. J Clin Invest 2008;118 (4):1311-1321.

2. Papadopoulos E, Gallidis E, Ptochos S. Anthelmintic resistance in sheep in Europe: A selected review. Vet Parasitology 2012;189(1):85-88.

3. Kaminsky R, Gauvry N, Schorderet WS, Skripsky T, Bouvier J, Wenger $A$, et al. Identification of the amino-acetonitrile derivative monepantel (AAD 1566) as a new anthelmintic drug development candidate. Parasitol Res 2008;103(4):931-939.

4. Kaplan RM. Drug resistance in nematodes of veterinary importance: a status report. Trends Parasitol 2004;20(10):477481.

5. Geary TG, Chibale K, Abegaz B, Andrae-Marobela K, Ubalijoro E. A new approach for anthelmintic discovery for humans. Trends Parasitol 2012;28(5):176-181.

6. Martin M, Mathias E, McCorkle CM. Ethnoveterinary Medicine: An annotated bibliography of community animal healthcare. 1rst ed. London, UK: ITDG Publising; 2001.
7. Bizimana N. Introduction. In: Traditional veterinary practice in Africa. 1rst ed. Rossdorf, Germany: Deutsche Gesellschaft fur Technische Zusammenarbeit (GTZ); 1994:8-13.

8. McGraw $\mathrm{U}$, Eloff JN: Methods for evaluating efficacy of ethnoveterinary medicinal. In: Katerere, DR, Luseba D editors. Ethnoveterinary botanical medicine. 1rst ed. USA: Taylor and Francis Group; 2010:1-24.

9. Waller PJ, Bernes G, Thamsborg SM, Sukura A, Richter $\mathrm{SH}_{\text {, }}$ Ingebrigtsen $\mathrm{K}$, et al. Plants as de-worming agents of livestock in the Nordic countries: historical perspective, popular beliefs and prospects for the future. Acta Vet Scand 2001;42:31-44.

10. Saika B, Borthakur SK. Use of medicinal plants in animal healthcare-A case study from Gohpur, Assam. Indian J Trad Knowledge 2010;9(1):49-51.

11. Tariq KA, Tantry MA. Preliminary studies on plants with anthelmintic properties in Kashmir-The north-west temperate Himalayan region of India. Chinese Medicine 2012;3:106-112.

12. Akhtar MS, Iqbal Z, Khan MN, Lateef M. Anthelmintic activity of medicinal plants with particular reference to their use in animals in the Indo-Pakistan subcontinent. Small Ruminant Res 2000;38(2):99-107.

13. Jain $P$, Singh $S$, Sandeep K, Verma SK, Kharya MD, Solanki S. Review: Anthelmintic potential of herbal drugs. International J Res Develop Pharmacy Life Sci 2013;2(3):412-427.

14. Prichard RK. Anthelmintic resistance. Vet Parasitol 1994;54(13):259-268.

15. Hoste $\mathrm{H}$, Jackson F, Athanasiadou S, Thamsborg SM, Hoskin SO. The effects of tannin-rich plants on parasitic nematodes in ruminants. Trends Parasitol 2006;22(6):253-261.

16. Lanusse $\mathrm{CE}$, Prichard RK. Relationship between pharmacological properties and clinical efficacy of ruminant anthelmintics. Vet Parasitol 1993;49(2-4):123-158.

17. Mottier L, Lanusse $C$. Bases moleculares de la resistencia a fármacos antihelmínticos. Rev Med Vet 2001;82(2):74-85.

18. Wink M. Medicinal plants: a source of anti-parasitic secondary metabolites. Molecules 2012;17(11):12771-12791.

19. Márquez LD. El desarrollo de nuevos antiparasitarios. En: Resistencia a los antihelmínticos en nematodos de rumiantes y estrategias para su control. 1rst ed. Colombia: Corpoica y Colciencias; 2007:24-25.

20. Fenwick A, Webster JP. Schistosomiasis: challenges for control, treatment and drug resistance. Curr Opin Infect Dis 2006;19(6):577-582.

21. McCarthy J. Is anthelmintic resistance a threat to the program to eliminate lymphatic filariasis? Am J Trop Med Hyg 2005;73(2):232-233.

22. Vercruysse J, Albonico M, Behnke JM, Kotze AC, Prichard RK. Is anthelmintic resistance a concern for the control of human soiltransmitted helminths? Int J Parasitol Drugs Drug Resist 2011;1(1):14-27.

23. Osei-Atweneboana MY, Awadzi K, Attah SK, Boakya DA, Gyapong JO, Prichard RK. Phenotypic evidence of emerging ivermectin resistance in Onchocerca volvulus. PLoS Negl Trop 2011;5:99-89.

24. WHO. World Health Organization. Accelerating work to overcome the global impact of neglected tropical diseases: a Roadmap for Implementation. Francia: WHO Press, 2012. 
25. Jabbar A, Iqbal Z, Kerboeuf D, Muhammad G, Khan MN, Afaq M. Anthelmintic resistance: the state of play revisited. Life Sci 2006;79(26):2413-2431.

26. Prichard RK, Hall CA, Kelly JD, Martin CA, Donald DA. The problem of anthelmintic resistance in nematodes. Aust Vet ] 1980;56(5):239-251.

27. Márquez DL. Resistencia a los antihelmínticos: origen, desarrollo y control. Revista Corpoica 2003;4(1):55-57.

28. Drudge JH, Leland SEJ, Wyant ZN. Strain variation in the response of sheep nematodes to the action of phenothiazine: II. Studies on pure infections of Haemonchus contortus. Am J Vet Res 1957; 18(67):317-325.

29. Reinemeyer CR. Diagnosis and control of anthelmintic-resistant Parascaris equorum. Parasit Vectors 2009;2(Suppl 2):1-6.

30. Craig TM. Anthelmintic resistance and alternative control methods. Vet Clin North Am Food Anim Pract 2006;22(3):567581.

31. Waller PJ, Prichard RK. Drug resistance in nematodes. In: Campbell y Rew editors. Chemotherapy of parasitic. 1rst ed. New York, USA: Plenum Press; 1986:339-362.

32. Prichard RK. Anthelmintic resistance in nematodes: extent, recent understanding and future directions for control and research. Int J Parasitol 1990;20:515-523.

33. Kaplan RM. Biology and management of anthelmintic resistance. In: Elsheikha HM, Khan NA editors. Essentials of veterinary parasitology. 1rst ed. Norfolk, UK: Caister Academic Press; 2011:201-204.

34. Waller PJ. International approaches to the concept of integrated control of nematode parasites of livestock. Int J Parasitol 1999;29(1):155-164.

35. Eddi C, Caracostantogolo J, Peña M, Schapiro J, Marangunich L, Waller PJ et al. The prevalence of anthelmintic resistance in nematode parasites of sheep in southern Latin America: Argentina. Vet Parasitol 1996;62(3-4):189-197.

36. Requejo-Fernandez JA, Martinez A, Meana A, Rojo-Vazquez FA, Osoro K, Ortega-Mora LM. Anthelmintic resistance in nematode parasites from goats in Spain. Vet Parasitology 1997;73(1):83-88.

37. Roeber F, Jex AR, Gasser RB. Impact of gastrointestinal parasitic nematodes of sheep, and the role of advanced molecular tools for exploring epidemiology and drug resistance-an australian perspective. Parasit Vectors 2013;6:153.

38. Van den Brom RL, Moll FH, Borgsteede M, Van Doorn DC, Lievaart-Peterson K, Dercksen DP et al. Multiple anthelmintic resistance of Haemonchus contortus, including a case of moxidectin resistance, in a Dutch sheep flock. Vet Record 2013;173(22):552

39. Nari A, Salles J, Gil A, Waller PJ, Hasen JW. The prevalence of anthelmintic resistance in nematode parasites of sheep in southern Latin America: Uruguay Vet Parasitol 1996;62(3-4):213222.

40. Echevarria F, Borba MF, Pinheiro AC, Waller PJ, Hasen JW. The prevalence of anthelmintic resistance in nematode parasites of sheep in southern Latin America: Brazil. Vet Parasitol 1996;62(34):199-206

41. Maciel S, Giménez AM, Gaona C, Waller PJ, Hansen JW. The prevalence of anthelmintic resistance in nematode parasites of sheep in southern Latin America: Paraguay. Vet Parasitol 1999;662(3-4):207- 212.

42. Van Wyk JA, Stenson MO, Van der Merwe JS, Vorster RJ, Viljoen PG. Anthelmintic resistance in South Africa: surveys indicate an extremely serious situation in sheep and goat farming. Onderstepoort. J Vet Res 1999;66(4):273-284.

43. Chandrawathani $\mathrm{P}$, Waller PJ, Höglud J. Evolution of high-level multiple anthelmintic resistance on a sheep farm in Malaysia. Trop Anim Health Prod 2003;35:17-25.

44. Chandrawathani P, Yusoff N, Wan LC, Ham A, Waller PJ. Total anthelmintic failure to control nematode parasites of smal ruminants on government breeding farms in Sabah, east Malaysia. Vet Res Commun 2004;28(6):479-489.

45. Mortensen LL, Williamson LH, Terril TH, Kircher RA, Larsen M, Kaplan RM. Evaluation of prevalence and clinical implications of anthelmintic resistance in gastrointestinal nematodes of goats. J Am Vet Med Assoc 2003;223(4):495-500.

46. Lacey $E$, Prichard RK. Interaction of benzimidazoles (BZ) with tubulin from BZsensitive and BZ-resistant isolates of Haemonchus contortus. Mol Biochem Parasitol 1986;19(2):171-181.

47. Lacey E. The role of the cytoskeletal protein tubulin in the mode of action and mechanism of drug resistance to benzimidazole. Int J Parasitol 1988;18(7):885-936.

48. Martin RJ. Modes of action of anthelmintic drugs. Vet J 1997; 154(1):11-34.

49. Moreno-Guzmán MJ, Coles GC, Jiménez-González A, Criadofornelio A, Ros-Moreno RM, Rodríguez-Caabeiro F. Levamisole binding sites in Haemonchus contortus. Int J Parasitol 1998;28(3):413-418.

50. Robertson AP, Bjorn $\mathrm{HE}$, Martin RJ. Resistance to levamisole resolved at the single-channel level. FASEB J 1999;13(6):749760.

51. Flemming JT, Squire MD, Barnes TM, Tornoe C, Matsuda K, Ahnn, et al. Caenorhabditis elegans levamisole resistance genes lev-1, unc-29, and unc-38 encode functional nicotinic acetylcholine receptor subunits. J Neuroscience 1997;17(15):5843-5857.

52. Arena JP, Liu KK, Paress PS, Schaeffer JM, Cully DF. Expression of a glutamate-activated chloride current in Xenopus oocytes with Caenorhabditis elegans RNA: evidence for modulation by avermectin. Brain Res Mol Brain Res 1992;8:339-348.

53. Cully DF, Vassilatis DK, Liu KK, Paress PS, Van der Ploeg LH, Schaeffer JM, Arena JP. Cloning of an avermectin-sensitive glutamate-gated chloride channel from Caenorhabditis elegans. Nature 1994;371:707-711.

54. Cully DF, Paress PS, Liu KK, Schaeffer JM, Arena JP. Identification of a Drosophila melanogaster glutamate-gated chloride canne sensitive to the antiparasitic agent avermectin. J Biol Chem 1996;271(33):20187-20191.

55. Laughton $\mathrm{DL}$, Lunt GL, Wolstenholme JA. Alternative splicing of a Caenorhabditis elegans gene produces two novel inhibitory aminoacid receptor subunits with identical ligand binding domains but different ion channels. Gene 1997;201(1-2):119-125.

56. Xu M, Molento M, Blackhall W, Ribeiro P, Beech R, Prichard RK Ivermectin resistance in nematodes may be caused by alteration of P-glycoprotein homolog. Mol Biochem Parasitol 1998;91(2):327-335. 
57. Molento MB, Prichard RK. Effects of multidrug-resistancereversing agents verapamil and $\mathrm{CL} 347,099$ on the efficacy of ivermectin or moxidectin against unselected and drug-selected strains of Haemonchus contortus in jirds (Meriones unguiculatus). Parasitol Res 1999;85(12):1007-1011.

58. Prichard RK. Drug resistance. Int J Parasitol 1999;29:137-138.

59. Jones AK, Buckingham SD, Sattelle DB. Chemistry-to-gene screens in Caenorhabditis elegans. Nat Rev Drug Discov 2005;4(4):321-330.

60. Moreno FC, Gordon IJ, Wright AD, Benvenutti MA, Saumell CA. Efecto antihelmíntico in vitro de extractos de plantas sobre larvas infectantes de nematodos gastrointestinales de rumiantes. Arch Med Vet 2010;42(3):155-163.

61. Githiori JB, Höglund J, Waller P, Baker L. Evaluation of anthelmintic properties of extracts from some plants used as livestock dewormers by pastoralist and smallholder farmers in Kenya against Heligmosomoides polygyrus infections in mice. Vet Parasitol 2003;118(3-4):215-226.

62. Pharmaceutical Society of Great Britain. Dept. of Pharmaceutical Sciences. British pharmaceutical codex. 10th ed. London: Pharmaceutical Press; 1973.

63. Mali RG, Mehta AA. A review on anthelmintic plants. Natural Product Radiance 2008;7(5):466-475.

64. Hammond JA, Fielding D, Bishop SC. Prospects for plant anthelmintics in tropical veterinary medicine. Vet Res Commun 1997;21(3):213-228.

65. Okpekon T, Yolou S, Gleye C, Roblot F, Loiseau P, Bories C et al. Antiparasitic activities of medicinal plants used in Ivory Coast. J Ethnopharmacol 2004;90(1):91-97.

66. Iqbal Z, Akthar MS, Sindhu ZU, Khan MN, Jabbar A. Review: Herbal dewormers in livestock-A traditional therapy. Int J Agr Bio 2003;5(2):199-206.

67. Waterman C, Smith RA, Pontiggia L, DerMaarderosian A. Anthelmintic screening of sub-Saharan African plant used in traditional medicine 2010;127:755-759.

68. Guarrera, PM. Traditional antihelmintic, antiparasitic and repellent uses of plants in central Italy. J Ethnopharmacol 1999;68(1):183192.

69. Bernt $U$, Junkersdorf $B$, Londershauesen $M$, Harder $A$, Schierenberg E. Effect of anthelminthics with different modes of action on the behavior and development of Caenorhabditis elegans. Fundam Appl Nematol 1998;21(3):251-263.

70. Harris TW, Nansheg C, Cunningham F, Tello-Ruiz M, Antoshechkin I, Bastiani C, et al. WormBase: A multi-species resource for nematode biology and genomics. Nucleic Acids Res 2004;32:411417.

71. Corsi AK. A biochemist's guide to C. elegans. Annal Biochem 2006;359(1):1-17.

72. Lacey E, Redwin JM, Gill JH, Demargheriti VM, Waller PJ. A larval development assay for the simultaneous detection of broad spectrum anthelmintic resistance. In: Boray JC, et al editors. Resistance of parasites to antiparasitic drugs. Rahway, NY, USA: MSD Agvet; 1990;177-184.

73. Dent JA, Davis MW, Avery L. Avr-15 encodes a chloride channel subunit that mediates inhibitory glutamatergic neurotransmission and ivermectin sensitivity in Caenorhabditis elegans. The EMBO J 1997;16(19):5867-5879.
74. Brenner S. The genetics of Caenorhabditis elegans. Genetics $1974 ; 77(1): 71-94$.

75. The $C$. elegans Sequencing Consortium. Genome sequence of the nematode $C$. elegans a platform for investigating biology. Science 1998,282:2012-2018.

76. Mitreva M, Zarlenga DS, McCarter JP, Jasmer DP. Parasitic nematodes - from genomes to control. Vet Parasitol 2007; 148(1):31-42.

77. 959 nematode genomes. http://www.nematodes.org/nematodegenomes/index.php/Species_published. Accessed Apr 15, 2015.

78. Geary TG, Thompson DP. Caenorhabditis elegans. how good a model for veterinary parasites? Vet Parasitol 2001;101(3-4):371386.

79. Driscoll ME, Dean E, Reilly E, Bergholz, Chalfie M. Genetic and molecular analysis of a Caenorhabditis elegans beta-tubulin that conveys benzimidazole sensitivity. J Cell Biol 1989;109(6 Pt 1): 2993-3003.

80. Trent $\mathrm{C}$, Tsuing N, Horvitz $\mathrm{H}$. Egg-laying defective mutants of the nematode Caenorhabditis elegans. Genetics 1983;104(4):619647.

81. Schafer W. Egg-laying. In: Jorgesen EM, Kaplan JM editors. USA: WormBook; 2005:1-7.

82. Zhang $\mathrm{M}$, Chung $\mathrm{SH}$, Fang-Yen $\mathrm{C}$, Craig C, Kerr RA, Suzuki $\mathrm{H}$ et al. A self-regulating feed-forward circuit controlling $C$. elegans egg-laying behavior. Curr Biol 2008;18(19):1445-1455.

83. Nematode.net (www.nematode.net).

84. Davis RE, Stretton AO. The motornervous system of Ascaris. Electrophysiology and anatomy of the neurons and their control by neuromodulators. Parasitol 1996;113:S97-S117.

85. Stretton AOW, Cowden C, Sithigorngul P, Davis RE. Neuropeptides in the nematode Ascaris suum. Parasitology 1991;102:107-116.

86. Kwa M, Veenstra J, Van Dijk M, Roos M. b tubulin genes from the parasitic nematode Haemonchus contortus modulate drug resistance in Caenorhabditis elegans. J Mol Biol 1995;246(4):500510.

87. Holden-Dye L, Walker RJ. Anthelmintic drugs. In: Villu M, Mclntire SL editors. USA: WormBook; 2007:1-13.

88. Lespine A, Ménez C, Bourguinat C, Prichard RK. P-glycoproteins and other multidrug resistance transporters in the pharmacology of anthelmintics: Prospects for reversing transport-dependent anthelmintic resistance. Int J Parasitol Drugs Drug Resist 2012;2:58-75.

89. McGaw $\sqcup$, Van der Merwe D, Eloff JN. In vitro anthelmintic, antibacterial and cytotoxic effects of extracts from plants used in South African ethnoveterinary medicine. Vet J 2007;173(2):366372.

90. Waterman C, Smith RA, Pontiggia L, DerMarderosian A. Anthelmintic screening of Sub-Saharan African plants used in traditional medicine. J Ethnopharmacol 2010;127(3):755-759.

91. Ndjonka D, Agyare C, Lüersen K, Djafsia B, Achukwi D, Nukenine $\mathrm{EN}$, et al. In vitro activity of Cameroonian and Ghanaian medicinal plants on parasitic (Onchocerca ochengl) and free-living (Caenorhabditis elegans) nematodes. J Helminthol 2011;85(3):304-312. 
92. Ndjonka D, Abladam ED, Djafsia B, Ajonina-Ekoti I, Achukwi MD, Liebau E. Anthelmintic activity of phenolic acids from the axlewood tree Anogeissus leiocarpus on the filarial nematode Onchocerca ochengi and drug-resistant strains of the free-living nematode Caenorhabditis elegans. J Helminthol 2014;88(4):481-488.

93. Katiki LM, Ferreira JF, Gonzalez JM, Zajac AM, Lindsay DS, Chagas $\mathrm{AC}$, et al. Anthelmintic effect of plant extracts containing condensed and hydrolyzable tannins on Caenorhabditis elegans, and their antioxidant capacity. Vet Parasitol 2013;192(1-3):218-227.

94. Katiki LM, Ferreira JF, Zajac AM, Masler C, Lindsay DS, Chagas $\mathrm{AC}$, et al. Caenorhabditis elegans as a model to screen plant extracts and compounds as natural anthelmintics for veterinary use. Vet Parasitol 2011;182(2-4):264-268.

95. Smith RA, Pontiggia L, Waterman C, Lichtenwalner M, Wasserman J. Comparison of motility, recovery, and methyl-thiazolyltetrazolium reduction assays for use in screening plant products for anthelmintic activity. Parasitol Res 2009;105(5):1339-1343.

96. Lai Y, Xiang M, Liu S, Li E, Che Y, Liu X. A novel high-throughput nematicidal assay using embryo cells and larvae of Caenorhabditis elegans. Exp Parasitol 2014;139:33-41.

97. Buckingham SD, Partridge FA, Sattelle DB. Automated, highthroughput, motility analysis in Caenorhabditis elegans and parasitic nematodes: Applications in the search for new anthelmintics. Int J Parasitol Drugs Drug Resist 2014;4(3):226-232.
98. Miltsch SM, Krücken J, Demeler J, Ramünke S, Harder A, von Samson-Himmelstjerna G. Interactions of anthelmintic drugs in Caenorhabditis elegans neuro-muscular ion channel mutants. Parasitol Int 2013;62(6):591-598.

99. Hu Y, Platzer EG, Bellier A, Aroian RV. Discovery of a highly synergistic anthelmintic combination that shows mutual hypersusceptibility. Proc Natl Acad Sci U S A 2010;107(13):59555960.

100. Peden AS, Mac P, Fei YJ, Castro C, Jiang G, Murfitt KJ, et al. Betaine acts on a ligand-gated ion channel in the nervous system of the nematode $C$. elegans. Nat Neurosci 2013;16(12):17941801.

101. Harder A, Schmitt-Wrede HP, Krücken J, Marinovski P, Wunderlich $\mathrm{F}$, Willson $\mathrm{J}$, et al. Cyclooctadepsipeptides-an anthelmintically active class of compounds exhibiting a novel mode of action. Int J Antimicrob Agents 2003;22(3):318-331.

102. Bull K, Cook A, Hopper NA, Harder A, Holden-Dye L, Walker RJ. Effects of the novel anthelmintic emodepside on the locomotion, egg-laying behaviour and development of Caenorhabditis elegans. Int J Parasitol 2007;37(6):627-636.

103. Burns AR, Luciani GM, Musso G, Bagg R, Yeo M, Zhang $Y$, et al. Caenorhabditis elegans is a useful model for anthelmintic discovery. Nat Commun 2015;6:7485. doi: 10.1038 /ncomms8485. 